Documentation et bibliothèques

DOCUMENTATION BIBLIOTHËUES

\title{
Musées et médias sociaux
}

Museums and Social Media

\section{Museos y medios de comunicación social}

\section{Sheila Carey}

Volume 55, numéro 4, octobre-décembre 2009

Muséologie et sciences de l'information

URI : https://id.erudit.org/iderudit/1029182ar

DOI : https://doi.org/10.7202/1029182ar

Aller au sommaire du numéro

\section{Éditeur(s)}

Association pour l'avancement des sciences et des techniques de la documentation (ASTED)

\section{ISSN}

0315-2340 (imprimé)

2291-8949 (numérique)

Découvrir la revue

Citer cet article

Carey, S. (2009). Musées et médias sociaux. Documentation et bibliothèques, 55(4), 177-187. https://doi.org/10.7202/1029182ar
Résumé de l'article

Le Web 2.0 (ou médias sociaux) transforme notre façon de communiquer. Les modèles de communication unilatérale cèdent le passage à la conversation. Les musées oeuvrent désormais dans un environnement au sein duquel chacun est libre de créer et de fournir de l'information sur un sujet, même sur un sujet pour lequel les musées faisaient figure d'autorité par le passé. En relevant les défis que pose ce nouvel environnement, les musées rejoignent non seulement les publics grâce aux applications Web 2.0, mais ils les invitent à participer à cet acte de co-création. De nombreux musées interagissent avec les publics par le biais des technologies Web 2.0, notamment Facebook, Flickr, YouTube et Second Life, en plus d'utiliser des applications de médias sociaux à partir de leurs propres sites. Le présent article porte sur les technologies Web 2.0 utilisées par les musées, sur les défis et les possibilités associés à ces technologies à des fins sociales.
Tous droits réservés (C) Association pour l'avancement des sciences et des techniques de la documentation (ASTED), 2009
Ce document est protégé par la loi sur le droit d'auteur. L'utilisation des services d'Érudit (y compris la reproduction) est assujettie à sa politique d'utilisation que vous pouvez consulter en ligne.

https://apropos.erudit.org/fr/usagers/politique-dutilisation/ 


\section{Musées et médias sociaux}

\section{SHEILA CAREY}

Analyste des audiences et des programmes Réseau canadien d'information sur le patrimoine sheila.carey@pch.gc.ca

\section{RÉSUMÉ | ABSTRACTS | RESUMEN}

Le Web 2.o (ou médias sociaux) transforme notre façon de communiquer. Les modèles de communication unilatérale cèdent le passage à la conversation. Les musées œuvrent désormais dans un environnement au sein duquel chacun est libre de créer et de fournir de l'information sur un sujet, même sur un sujet pour lequel les musées faisaient figure d'autorité par le passé. En relevant les défis que pose ce nouvel environnement, les musées rejoignent non seulement les publics grâce aux applications Web 2.0, mais ils les invitent à participer à cet acte de co-création. De nombreux musées interagissent avec les publics par le biais des technologies Web 2.o, notamment Facebook, Flickr, YouTube et Second Life, en plus d'utiliser des applications de médias sociaux à partir de leurs propres sites. Le présent article porte sur les technologies Web 2.0 utilisées par les musées, sur les défis et les possibilités associés à ces technologies à des fins sociales.

\section{Museums and Social Media}

Web 2.0 or social media changes the way we communicate. The unidirectional communication models are being replaced with bidirectional or conversational models. Museums currently operate in an environment in which each person is free to create and supply information about a subject, even about one for which the museum was the recognised authority. In responding to these new challenges, museums can now better reach the public with Web 2.0 applications and can now invite the public to collaborate in the creative process. Several museums interact with their publics, namely with the help of Web 2.0 technologies such as Facebook, Flickr, YouTube and Second Life as well as with the social media applications on their web sites. This article describes the Web 2.0 technologies used by museums as well as the challenges and opportunities these technologies can bring to society.

\section{Museos y medios de comunicación social}

La Web 2.o (o medios de comunicación social) transforma nuestra manera de comunicarnos. Los modelos de comunicación unilateral ceden el paso a la conversación. En la actualidad, los museos se desenvuelven en un entorno en el que cada uno es libre de crear $y$ de proporcionar información, incluso sobre temas para los cuales los museos constituían una figura de autoridad. Al aceptar los desafíos que plantea este nuevo entorno, los museos no sólo reúnen público, gracias a las aplicaciones Web 2.o, sino que también lo invitan a participar en este acto de creación compartida. Muchos museos interactúan con el público por medio de las tecnologías Web 2.o, en especial, Facebook, Flickr, You Tube y Second Life, además de utilizar aplicaciones de medios de comunicación social en sus propios sitios. Este artículo abarca las tecnologías Web 2.0 implementadas por los museos, así como los desafíos y las posibilidades relacionados con estas tecnologías con fines sociales.

\section{Introduction}

I 'Utilisation D'Internet dans les musées a grandement évolué depuis les années 1990. Vers 1995, les musées se contentaient de créer des sites qui étaient en somme des "brochures " publicitaires puisqu'à cette époque, Internet était un réseau publiant en général de l'information dans des sites Web ou permettant des interactions personnelles au moyen du courriel.

Dans l'intervalle et jusqu'en 2009, non seulement de plus en plus de gens se sont branchés au réseau, mais les données démographiques sur les internautes ont changé, la quantité de contenu en ligne a augmenté de façon exponentielle et les types d'interactions ont changé au fur et à mesure de lévolution des technologies rendant ces interactions possibles.

Selon un rapport de Pew Internet, moins de $15 \%$ des adultes américains étaient en ligne en mai-juin 1995, comparativement à près de $75 \%$ en décembre $2008^{1}$ (<http://www.pewinternet.org/Data-Tools/DownloadData/Trend-Data.aspx $>$ ).

Les données démographiques liées à l'utilisation virtuelle ont également changé au fil du temps, et le réseau que l'on croyait principalement utilisé par les jeunes a élargi son éventail d'utilisateurs avec plus de la moitié des internautes âgés entre 18 et 44 ans et de plus forts pourcentages d'utilisateurs en ligne plus âgés que par le passé (Jones et Fox, 2009).

À l'heure actuelle, les gens engagent des conversations en ligne et ils s'attendent à pouvoir communiquer leurs idées et opinions sur un vaste éventail de sujets. À mesure que l'accès à l'information façonne les changements, les musées doivent également continuer à réinventer leur présence sur la toile afin de suivre l'évolution et de répondre aux attentes des visiteurs en ligne. Dans certaines situations, les musées ont reconnu la nécessité d'aller de l'avant et de fournir de l'information dans les espaces en ligne fréquentés par les visiteurs. Dans d’autres cas, les musées offrent aux visiteurs la possibilité de s'exprimer à partir du site même du musée. Cependant, ils doivent le faire dans le contexte de l'etablissement et le respect de son rôle, et en s'efforçant de préserver l'image et l'identité que le musée tente de projeter. C'est une ques-

1. Pour 1995, les statistiques indiquaient un accès aux babillards électroniques et aux services d'information; à partir de 2005 , les chiffres comprennent les utilisateurs qui utilisent Internet pour accéder aux sites Web ou au courrier électronique. 


\section{La perte de contrôle est la plus grande crainte des professionnels des musées à l'égard des médias sociaux puisque, pendant de nombreuses années, les musées étaient seuls maîtres $d u$ contenu et du message.}

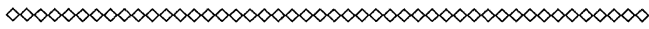

tion déquilibre pour les musées entre l'obligation d'emboîter le pas aux changements relatifs à la technologie et aux attentes, tout en étant fidèle au rôle premier du musée.

Le Conseil international des musées (ICOM) définit le musée comme "une institution permanente sans but lucratif, au service de la société et de son développement, ouverte au public, qui acquiert, conserve, étudie, expose et transmet le patrimoine matériel et immatériel de l'humanité et de son environnement à des fins d'études, d'éducation et de délectation" (Statuts de l'ICOM, article 3, paragraphe 1). Dans le cadre de lévaluation des possibilités offertes par le Web 2.0, les musées doivent évaluer la mesure selon laquelle ces applications cadrent avec leurs responsabilités; en règle générale, l'utilisation des médias sociaux par les musées est harmonisée à la recherche et à la communication aux fins d'éducation, détudes et de délectation.

Depuis toujours, les musées ont le mandat de créer du contenu reconnu et on les considère comme des sources d'information fiables (Griffiths et King, 2008). Par conséquent, de quelle façon les musées arriventils à suivre cette évolution, alors qu'ils sont tenus de conserver une certaine pertinence pour les publics qui saattendent (et même demandent) une interactivité et un droit de parole, tout en préservant l'autorité et l'identité du musée?

La perte de contrôle est la plus grande crainte des professionnels des musées à légard des médias sociaux puisque, pendant de nombreuses années, les musées étaient seuls maitres du contenu et du message (Simon, 2008a). Les conservateurs, qui ont consacré des années à la recherche et au développement d'information officielle au sujet des collections muséales, peuvent parfois considérer les discussions dans les médias sociaux comme une menace à leur pouvoir de conservation (Ferrante, 2009). Simon suggère que les musées ont raison de vouloir protéger l'expertise de leur personnel et celle qui transparait dans leurs expositions, leurs programmes et leurs collections, mais ils ne devraient pas se sentir autorisés à contrôler l'expérience du visiteur. La question nest pas . de donner tous les pouvoirs aux visiteurs, mais de leur offrir des possibilités de créer leurs propres messages et de classer par ordre de priorité ceux qui leur conviennent le mieux (Simon, 2008a). Toutefois, Simon insiste sur le fait que, avec les médias sociaux, on a toujours le pouvoir détablir les règles régissant le comportement et l'utilisation, d'exploiter le contenu produit par les utilisateurs, de faire la promotion et de présenter le contenu retenu, et de définir les interactions possibles. De tels pouvoirs permettent au musée de conserver un certain degré de contrôle.

Avec lévolution de la technologie, qui a permis de créer des présentations en ligne plus élaborées, les musées sont passés de lélaboration de simples "brochures" en ligne à la création d'expositions virtuelles sophistiquées comme façon de présenter le contenu en ligne conservé et délargir la portée du contenu hors des murs du musée. Certains musées ont également commencé à offrir de l'information en ligne sur les collections, malgré les préoccupations exprimées par d'autres musées qui craignaient que cela ne dissuade les visiteurs de se rendre au vrai musée et touche, par ricochet, le bénéfice net de ce dernier. Certaines études ont toutefois révélé que le contenu en ligne ne semblait pas détourner les visites à létablissement et qu'il pouvait même inciter les visiteurs à se rendre sur place (Griffiths et King, 2008; RCIP, 2005).

\section{Aller de l'avant : présenter le contenu hors du site du musée}

Avec les premiers sites Web, la plupart des musées avaient atteint un certain degré de confort dans la présentation du contenu dans une "arène " différente que l'établissement muséal en tant que tel. Aujourd'hui, ils doivent relever de nouveaux défis afin de combler les attentes des utilisateurs en ligne et de rejoindre ces utilisateurs dans les sites qu'ils fréquentent; c'est pourquoi les musées ont commencé à présenter leur contenu dans d'autres sites que les leurs.

Les musées se tournent vers des sites de l'extérieur dans le but délargir leur communauté virtuelle. Dans certains cas, ils ont créé ce que l’on pourrait appeler des "brochures virtuelles » de deuxième génération, présentées dans des sites comme Facebook ou MySpace. Ils ont parfois poussé plus loin l'expérience en présentant un contenu enrichi, des images de leurs collections ou leur programmation par exemple, dans des sites de partage d'images ou de vidéos comme Flickr ou YouTube.

\section{Facebook}

Dans le cas des musées présents dans Facebook, les pages servent principalement à rejoindre la communauté dans le but détablir de nouvelles relations en ligne ou d'approfondir les relations existantes au sein d'une communauté. En plus d'ajouter dans leurs pages Facebook de nouveaux éléments, des événements par exemple, les musées y présentent également des photographies et des vidéos.

Même si l'information présentée s'apparente à celle publiée dans les premiers sites Web des musées, 
en diffusant cette information dans les espaces publics, le musée abandonne une certaine part de contrôle puisque les visiteurs peuvent y écrire ce qu'ils veulent. Les membres de Facebook peuvent donc formuler des commentaires en ligne au sujet d'un musée (ou même créer une "Fan Page» pour le compte du musée) et créer des représentations en ligne pour un musée, sans la participation de ce dernier.

Bien que certains musées puissent voir en Facebook une façon d'attirer de nouveaux publics plus jeunes, des études réalisées récemment ont révélé que Facebook et d'autres espaces sociaux gagnent en popularité auprès d'autres groupes d'âges (Lenhart, 2009; Nielsen, 2009). Alors que Facebook était à ses débuts un service destiné aux étudiants des universités, presque un tiers de l'ensemble de ses membres sont maintenant âgés de 35 à 49 ans et près du quart ont plus de 50 ans (Nielsen, 2009). Ces chiffres indiquent que, bien que Facebook ne soit pas l'espace idéal pour cibler les jeunes et qu'il ne représente plus le créneau pour lequel il était reconnu au départ, il demeure une avenue intéressante pour les musées prêts à diffuser de l'information à jour. Les utilisateurs de médias sociaux, notamment des blogues, de Facebook et de Twitter, ont des attentes relatives à la fiabilité de l'information; les établissements doivent donc prendre soin détablir des attentes réalisables
Les utilisateurs de médias sociaux, notamment des blogues, de Facebook et de Twitter, ont des attentes relatives à la fiabilité de l'information.

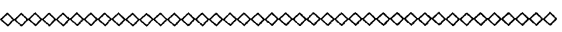

lorsqu'ils entreprennent de travailler avec ces médias sociaux (Marsan, 2009).

Certains musées ont mis à profit les médias sociaux dans le cadre de leurs campagnes de financement. À titre d'exemple, le Brooklyn Museum (<http://www.brooklynmuseum.org/>), par le réseau " $1^{\text {st }}$ Fans ", offre à ses membres des communautés virtuelles du musée, notamment Facebook, Flickr et Twitter, de recevoir des invitations pour les événements du premier samedi du mois. Ces membres virtuels nont pas les mêmes avantages qu'un membre à part entière du musée, mais ils ont un sentiment d'appartenance attribuable au fait qu'ils font partie d'une communauté (Cary, 2008).

Il est trop tôt pour déterminer si de telles initiatives réussiront à maximiser les avantages, mais il est important de reconnaitre que même les méthodes classiques, comme ladhésion, affichent le potentiel délargir les possibilités dans la sphère virtuelle.

\section{Figure 1}

1stfans, Musée de Brooklyn (New York).

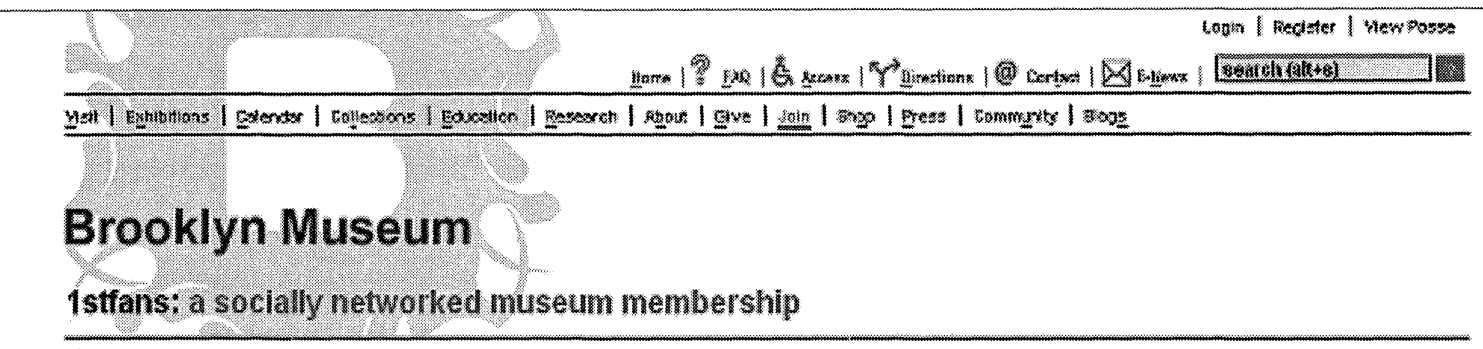

sodalize at exclusive meetups during the Museum's monthly Target First Saturdays and continue comnecting online with access to artistcrealed content on our 1strans Twitter Art Feed. This paperless Membership is only s20 for the year and is fully tex-deductible!

When you become a 1stfan, you can:

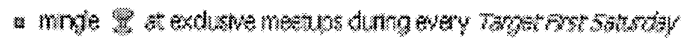

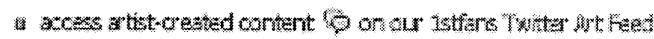

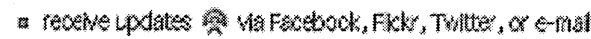

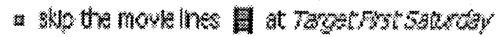

join 1stfans!

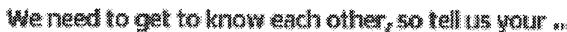
N.sman

\section{1 stfans introduction}

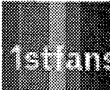

read Will Carys introduction to Istans

\section{1stfans blog}

Istrans Witter Art Feed Antist for August 200s: Lauren Mecarthy The Istlone Twitter AI Feed antist or August is Lau en hocarth, a young a tst who has aready wom many hats. A sell-desctibed "eitistprogratmer resigneworson: La inen's work explc es the itargestion of physical yaut wme.

Istrans Twitter Art Feed Artist for July 2009: Ranjit Ehatnagar's "Exquisite sonnet" Judghy oy the nunver

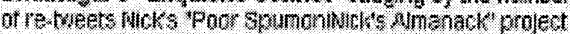
teceked, think ths sate to soy that I stans enoy Twilte At Feed oro ats that nix lancuase and wit rend mur. 


\section{Divers établissements pourraient ne pas être à l'aise avec le fait que leur contenu se retrouve dans les sites de tierces parties et avec les commentaires des utilisateurs formulés dans de tels sites.}

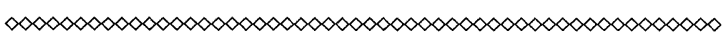

\section{YouTube}

Certains musées ont optimisé leur portée dans les communautés virtuelles en créant une chaîne dans YouTube. Le Centre des sciences de l'Ontario (CSO) (<http://www.centredessciencesontario.ca/default.asp $>$ ) a téléchargé un contenu vidéo non seulement dans YouTube, mais aussi dans 19 sites différents - d'Atom Uploads, à Exposure $\mathrm{CBC}$, en passant par Yahoo! Canada Video - et ces 340 vidéos réalisées par le CSO ont été vues plus de cinq millions de fois (von Appen et coll., 2009). À partir de ces sites, les visiteurs peuvent visionner les vidéos, y répondre, leur accorder une note et formuler des commentaires à leur sujet. C'est une vitrine supplémentaire pour le contenu du musée, une ouverture vers un public plus vaste, avec en arrière-plan le risque de perte de contrôle que craignent certains musées.

Le CSO a poussé plus loin l'expérience en organisant une rencontre YouTube, le 8 août 2008, qui a réuni des visiteurs du monde entier dans le cadre d'un événement au CSO (la rencontre 888 à Toronto). Des participants provenaient d'aussi loin que l'Australie, l'Argentine, le Royaume-Uni et les États-Unis, mais la rencontre a également attiré son lot de jeunes de la région. Apparemment, cétait la première rencontre organisée et tenue dans un musée des sciences, et la plus importante à ce jour, réunissant au musée 460 participants (von Appen et coll., 2009).

Une étude de Alexander et coll. (2008) suggère fortement que la diffusion vidéo d'un contenu dans YouTube ne suffit pas à provoquer l'achalandage, physique ou virtuel, au musée. Des preuves convaincantes (Bernstein, 2008) suggèrent qu'un contenu créé par le visiteur peut être plus attrayant et mieux cadrer avec la dynamique de la communauté de YouTube que le contenu produit par le musée. L'expérience de la rencontre du 888 confirme ces conclusions et suggère qu'il serait bon d'exploiter lorganisation d'autres rencontres, et d'envisager des rencontres de collaboration entre les musées, afin d'attirer de nouveaux publics par de nouvelles voies (von Appen et coll., 2009).

L'expérience observée par Alexander et d'autres chercheurs dans le cadre d'un projet de recherche dans YouTube englobant cinq musées a révélé que les musées considéraient qu'il était avantageux pour leur établisse- ment d'y participer. Cependant, il était difficile de quantifier cet avantage, et cétait souvent par le biais d'information qualitative, notamment les commentaires, que les musées concluaient qu'il était avantageux de faire partie d'une communauté de partage de vidéos (Alexander et coll., 2008).

Alors que certains établissements et leur personnel, à l'instar de Peter Foley du Museum of Modern Art de New York (MoMA), apprécient le plaisir de faire découvrir leur contenu par les utilisateurs, certains demeurent prudents à l'égard de YouTube et s'inquiètent du fait de donner au public un accès illimité au contenu, prônant que les musées doivent faire attention à ce qui est diffusé. Parmi les aspects à examiner, mentionnons le fait que les autorisations et les droits d'auteur doivent être respectés et qu'il ne faudrait pas, par exemple, que des séquences en coulisse ou du métrage nuisent à la sécurité (Yasko, 2007). En outre, les musées doivent savoir que leur contenu pourrait être utilisé sans autorisation ou hors contexte. Divers établissements pourraient ne pas être à l'aise avec le fait que leur contenu se retrouve dans les sites de tierces parties et avec les commentaires des utilisateurs formulés dans de tels sites. Certains musées surveilleront ou mitigeront les commentaires publiés dans ces sites, tandis que d'autres seront davantage enclins à laisser les utilisateurs s'exprimer à leur aise, en fonction de leurs politiques institutionnelles.

\section{Flickr}

Flickr est un autre site dans lequel les musées ont téléchargé du contenu. Flickr est un service qui permet aux utilisateurs de télécharger des images, de les annoter et de les partager. Parmi les annotations possibles, on compte les balises géographiques, ce qui fait de Flickr une ressource très pratique pour les utilisateurs qui veulent créer des applications composites qui permettent aux images dêtre jumelées à d’autres applications, comme par exemple les cartes de Google. Plusieurs musées ont téléchargé du contenu dans Flickr. Le Brooklyn Museum a fait figure de pionnier dans l'utilisation des nombreuses formes de médias sociaux, et il a été l'un des premiers musées à recourir à Flickr lorsqu'il a créé un espace pour les visiteurs afin qu'ils publient leurs propres images de graffiti dans le cadre d'une exposition du musée intitulée "Graffiti » (<http://www.flickr.com/ photos/brooklyn_museum/sets/72157594171809720/>).

Plusieurs autres musées ont fait l'expérience de publier des images dans Flickr afin de vérifier quel usage feraient les utilisateurs de leur contenu, et quels types d'annotations y seraient ajoutées. La fonction d'annotation, sur laquelle nous reviendrons plus loin, est une caractéristique commune aux médias sociaux découlant du désir des utilisateurs détiqueter les articles afin d'y revenir ultérieurement. Del.icio.us est sans doute le système d'annotation le plus répandu, mais d'autres, comme Digg, offrent la même fonction; en étiquetant 
un contenu, l'utilisateur peut le récupérer facilement, mais il devient aussi plus facile pour d'autres utilisateurs affichant les mêmes intérêts de repérer ce même contenu.

En 2006, Pete Gray, qui œuvrait alors pour les musées d'East Lothian en Écosse, a commencé à utiliser Flickr pour y présenter des images et des données sur les collections. Il avait décidé que cétait un moyen simple et peu cher pour les musées de présenter en ligne leurs collections. Il a tout simplement ajouté des images à l'information disponible et placé le tout dans Flickr pour voir ce qui se passerait. Les images dans Flickr ont été reproduites dans d'autres espaces (dans des blogues) de sorte qu'il a pu observer la multiplication des utilisations des images publiées dans Flickr (McKenzie, 2008). C'est l'un des avantages associés à la création de contenu destiné aux médias sociaux : le contenu peut être réutilisé de plusieurs façons par le truchement de diverses applications.

Le 16 janvier 2008, la Library of Congress (Bibliothèque du Congrès) aux États-Unis a lancé un projet dans Flickr (<http://www.flickr.com/photos/library_ of_congress/collections/72157601355524315/>). Le site de partage Flickr avait été reconnu comme le lieu de présentation en ligne répondant à toutes les exigences établies - un espace où télécharger des images, une communauté active axée sur les images dotée de fonctions de balisage, de partage et de formulation de commentaires - sauf à celle liée aux droits d'auteur. Aucune des options disponibles en matière de licence ne convenait à un établissement culturel qui nétait pas l'initiateur ou le créateur des œuvres (Springer et coll., 2008). Des discussions avec Flickr ont mené à l'utilisation de l'énoncé : «Aucune restriction de droit d'auteur connue " qui est désormais disponible pour les autres établissements culturels publiant dans The Commons.

Dans le cadre de son projet dans Flickr, la Library of Congress a publié deux ensembles comportant environ 3 ooo photographies historiques à l'adresse $<\mathrm{http}$ ://www. flickr.com/photos/library_of_congress $>$, et a invité le public à annoter les images en publiant le message : « La Bibliothèque du Congrès demande l'aide du public ». Les objectifs du projet étaient les suivants :

- accroître la sensibilisation par le partage de photographies issues des collections de la Library of Congress avec des gens qui apprécient les images mais n'auraient probablement pas visité son site Web ;

- mieux comprendre en quoi les annotations du public et l'apport de la communauté pourraient être avantageux à la fois pour la Library of Congress et les utilisateurs des collections ;

- acquérir de l'expérience en participant aux communautés émergentes dans le Web qui pourraient avoir un intérêt pour le genre de documents qui constituent les collections de la Library of Congress (Springer, 2008).
Le personnel de la bibliothèque vérifiait l'information fournie puis l'intégrait aux enregistrements de son catalogue, ce qui a contribué à enrichir les renseignements disponibles pour certaines des images.

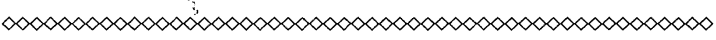

Les images ont été visionnées par de très nombreux internautes; il y eut 1,1 million de consultations dans les premières 24 heures après la diffusion, et 3,6 millions de consultations après une semaine. Au moment de la publication du rapport, plus de 4 ooo photos avaient été publiées (Springer et coll., 1988). En date du 23 octobre 2008, on a pu confirmer les statistiques suivantes:

- 10,4 millions de consultations des photos dans Flickr;

- $79 \%$ des 5615 photos avaient été ajoutées dans les favoris (c'est-à-dire ajoutées aux collections personnelles dans Flickr);

- plus de 15000 membres de Flickr avaient choisi d'ajouter comme contact la Library of Congress, créant un album de photos des images de la Library dans leurs propres comptes ;

- 4548 des 4615 photos avaient reçu au moins une annotation fournie par un membre de la communauté ;

- 67176 annotations ont été ajoutées par 2518 comptes uniques de Flickr; moins de 25 cas de contenu non approprié créé par des utilisateurs ont été retirés (Springer et coll., 2008).

Les internautes disposent de trois façons d'ajouter de l'information dans Flickr : les commentaires à structure libre, les annotations et les notes appliquées aux éléments des images mêmes. Les commentaires prenaient souvent la forme de souvenirs, d'identifications et de corrections. Les photos recevaient de nouvelles descriptions, de nouveaux mots-sujets, des réponses émotives, des renseignements personnels et diverses formes de mots existants. Le personnel de la bibliothèque vérifiait l'information fournie puis l'intégrait aux enregistrements de son catalogue, ce qui a contribué à enrichir les renseignements disponibles pour certaines des images. L'examen des annotations a révélé que, sur les 59193 annotations ajoutées jusquau 13 mai 2008, $40 \%$ avaient été ajoutées par un petit groupe de 10 marqueurs très actifs qui ont fourni plus de 3 ooo annotations (Springer et coll., 2008). Un autre groupe d'environ 20 commentateurs très actifs également visitait régulièrement le site et fournissait des renseignements appropriés en vue de corriger le lieu, de préciser les dates ou le nom des événements, tout en appuyant souvent ses dires par des liens menant aux archives du New York Times, à Wikipedia et à des sites Web spécialisés et dédiés à des zones d'intérêt en particulier (Springer et coll., 2008). 
Les musées qui partagent des images à partir de Flickr doivent décider du contenu qu'ils sont prêts à partager, à quel cồt et quel niveau d'engagement ils sont prêts à assumer.

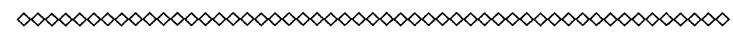

La Library of Congress a également observé une augmentation des activités dans son propre site, ce qui a contribué à accroître son degré d'exposition en ligne. Le coût permanent du projet pour l'établissement, y compris la gestion du compte et l'ajout de nouvelles images, correspond à un employé équivalent temps plein; une participation plus active nécessiterait des ressources supplémentaires.

La Library of Congress est un établissement public réputé et elle a bénéficié d'une forte publicité positive pour son projet pilote par le truchement de blogues et grâce à une couverture des médias. Le personnel a eu limpression que le projet a fait vibrer la corde de l'altruisme au sein de la communauté lorsqu'il a demandé l'aide des membres dès l'introduction du projet; il est possible que cette bonne volonté, conjuguée au prestige de létablissement, ait contribué à l'atteinte des résultats favorables du projet. Puisque la Library of Congress était le premier établissement à introduire un tel projet, les résultats pourraient être plus mitigés lorsque l'approche sera appliquée à grande échelle. Toutefois, les amateurs forment l'un des principaux publics d'Internet (Griffith, 2007), et ce type de projet (selon les commentateurs actifs) pourrait interpeller ce public en particulier.

La réaction positive au projet a incité Flickr à lancer The Commons (www.flickr.com/commons) et à inviter les organismes culturels patrimoniaux à participer. Les musées qui publient du contenu dans Flickr s'inquiètent de la perte de contrôle relativement aux droits d'auteur. Bien qu'il y ait des indicateurs précis pour les droits d'auteur, il nest pas possible sur le plan technique de s'assurer que les gens respectent de tels droits ; l'information y est donc diffusée de bonne foi. Par conséquent, les œuvres les plus précieuses, régies par des règles rigoureuses sur le droit d'auteur, nont pas leur place dans des sites de partage d'images comme Flickr.

Le Powerhouse Museum de Sydney ( $<$ http://www. powerhousemuseum.com/ $/$ ), à linstar du Brooklyn Museum, sest taillé une réputation détablissement novateur en ligne et, comme le Brooklyn Museum, il est également un chef de file de la communauté non seulement par sa participation à des projets novateurs, mais par la diffusion des résultats de ses projets dans des blogues et lors de conférences telles Museum and the Web $(<\mathrm{http} / / /$ www.archimuse.com/conferences/mw.html $>$ ). Le Powerhouse Museum a mis en œuvre l'annotation de l'information sur ses collections dans son site en 2006 (Chan,
2006, 2007) et a produit de nombreux rapports sur ce projet. Après avoir observé le travail de l'extension The Commons de Flickr, le personnel a décidé d'importer des images dans The Commons, de faire le suivi des réactions et d'analyser les types d'annotations obtenues en rapport au contenu (Chan, 2008a). Il a répertorié les annotations qui ont été appliquées dans Flickr et les a intégrées à son système de gestion des collections. Entre le 8 avril 2008 et le 8 avril 2009, le Powerhouse Museum a téléchargé 1171 photos dans Flickr (382 avec balises géographiques) issues de quatre collections photographiques d'archives différentes. Durant cette période, les photos ont été visionnées 777466 fois (Chan, 2009). Le personnel considère que l'expérience est concluante puisque les photographies n'avaient jamais été aussi utilisées dans le site même du musée. De plus, le Powerhouse Museum a pu tirer parti de l'information recueillie et d'identifications supplémentaires, d'applications composites et de nouvelles œuvres, etc., découlant de la participation de la communauté.

Les 200 images ajoutées à lorigine et provenant de la collection de photos Tyrrell du Powerhouse Museum (l'une des quatre collections photographiques d'archives téléchargées dans Flickr par le Powerhouse Museum) étaient vendues précédemment sous forme d'images numériques et on leur attribuait une grande valeur. Elles étaient accessibles à partir du site Web du Powerhouse Museum depuis plusieurs années, mais pas dans un format téléchargeable. La diffusion de telles images dans une plus vaste communauté était une entreprise difficile pour les établissements de collecte dotés d'une politique d' « accès sur paiement». En se fondant sur les statistiques recueillies sur la vente et les demandes liées aux images de la collection Tyrrell, on a établi qu'il n'y avait aucun effet nuisible sur les recettes découlant des ventes; au contraire, jamais les images n'avaient bénéficié d'une telle vitrine avant leur exposition dans The Commons. Les résultats obtenus grâce à ce projet ont incité le Powerhouse Museum à modifier son modèle classique de licence relative aux images et à concevoir un nouveau modèle commercial afin de garantir un meilleur accès au contenu numérique (Bray, 2009). Les projets comme celui-là permettent aux musées de proposer un projet à petite échelle, d'examiner les résultats et de concevoir de nouveaux modèles commerciaux. Le Powerhouse Museum envisage d'introduire des options en matière d'impression d'ouvres d'art semblables à celles offertes par le service en ligne du Victoria and Albert Museum (<http://www.vam.ac.uk/>) et il reconnait que de telles possibilités doivent être étudiées dans le cadre des prochains projets liés au partage d'images (Bray, 2009).

Les musées qui partagent des images à partir de Flickr doivent décider du contenu qu'ils sont prêts à partager, à quel coût et quel niveau d'engagement ils sont prêts à assumer (Simon, 2008b). Ils doivent également décider si lajout de contenu dans des sites externes permet réellement détablir des relations suffisantes avec 
les utilisateurs qui peuvent parcourir le contenu sans réaliser la valeur de ce contenu provenant du musée. Avec Facebook, il est plus évident que le musée crée une communauté et qu'il développe sa relation avec ses " amateurs "; avec Flickr, rien ne prouve que ceux qui le consultent choisissent ce contenu particulièrement parce qu'il provient du musée. Bernstein a fait remarquer qu'il est important, lorsque lon a recours à des fonctions des médias sociaux, de créer une communauté et de ne pas simplement utiliser les espaces communautaires comme outils de marketing; le Brooklyn Museum a créé une application qui permet aux utilisateurs de Facebook de choisir des œuvres d'art de sa collection et de les utiliser dans leurs pages Facebook (Bernstein, 2009). Le Brooklyn Museum, en intégrant de nombreuses fonctions à son profil Facebook, comme par exemple le flux RSS, et en reliant le contenu existant dans d'autres sites comme Flickr, del.icio.us, YouTube et son blogue, a su se servir de Facebook pour créer un autre site Web plutôt qu'une simple brochure publicitaire.

Que ce soit par une présence dans Facebook, MySpace, par un flux Twitter ou un contenu téléchargé dans Flickr ou YouTube, en règle générale, les musées participent à ces espaces pour sensibiliser les publics et élargir leur portée dans la communauté. Les avantages associés à de tels espaces sont nombreux, notamment la portée mondiale et l'atteinte de communautés voisines en accédant à plusieurs plateformes (Simon, 2008b).

\section{Annotations du contenu muséal par les utilisateurs}

Certains projets ont étudié les annotations publiées dans un site muséal par les utilisateurs. Les annotations créées par le public, ou folksonomies, constituent des données de classification décentralisées produites par le public. Les projets sur les annotations dans les systèmes muséaux sont une réponse à l'utilisation changeante d'Internet au fil du temps. Les systèmes de gestion des collections de musées sont conçus afin de documenter les collections d'un musée pour les besoins internes et ils emploient une terminologie particulière aux professionnels de musées. Lorsqu'ils diffusent leurs collections en ligne, ils donnent accès au public à ces collections. Toutefois, les utilisateurs ne sont pas toujours capables de trouver les enregistrements des collections parce qu'ils n'emploient pas la terminologie des spécialistes. Dans le cadre de certains projets, on a tenté de combler cet écart entre la terminologie des professionnels des musées et le vocabulaire général des chercheurs en ligne; le Réseau canadien d'information sur le patrimoine (RCIP), par exemple, se sert du Art \& Architecture Thesaurus (AAT) comme assistant de recherche au sein d'Artefacts Canada (<http://Pro-Ex/chin.gc.ca/normes-standards/recherches_courant-current_research-fra.jsp $>$ ).

Les annotations par les visiteurs sont une autre façon de tenter de combler ce fossé de communication.

\section{Dans le cadre de certains projets, on a tenté de combler cet écart entre la terminologie des professionnels des musées et le vocabulaire général des chercheurs en ligne.}

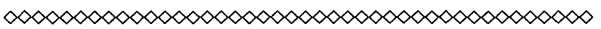

Toutefois, la question se pose sur le nombre d'utilisateurs qui annoteront le contenu en ligne des musées de façon altruiste, pour le seul plaisir de le faire (Chan, 2007). Plusieurs études ont été réalisées à ce sujet par la communauté Interaction personne-machine. La recherche suggère que le fonctionnement des systèmes sociaux d'annotations repose en général sur de grands groupes d'utilisateurs et que le taux de participation est partiellement motivé par une interaction et une communication exigeant peu d'efforts (Budiu et coll., 2009). Le jeu ESP (<http://www.gwap.com/gwap/gamesPreview/ espgame/>) a été l'une des premières initiatives visant à encourager les annotations par le public, tout en offrant un incitatif aux participants (von Ahn et Dabbish, 2004). Dans le cadre de ce jeu, les visiteurs annotaient les images sans réaliser le but sous-jacent. En comparant les annotations des participants au jeu aux étiquettes attribuées par les personnes à qui l’on avait demandé de décrire les images, on a découvert que pour la totalité des images, au moins cinq ( $83 \%$ ) des six étiquettes produites dans le cadre du jeu étaient identiques à celles des autres participants. Cette première initiative avait réussi à trouver une façon d'inciter les utilisateurs à produire des annotations en créant une application populaire.

Dans le cadre du projet de musée Steve $(<\mathrm{http}: / /$ www.steve.museum/>), on a effectué des études approfondies sur les annotations propres au contenu en ligne d'une galerie d'art. Le rapport le plus récent porte sur la recherche réalisée entre octobre 2006 et décembre 2008. L'idée consistait à améliorer l’accès aux collections en ligne en demandant aux utilisateurs de proposer des termes d'un vocabulaire plus populaire que ceux utilisés par la communauté des musées afin de décrire les collections. Voici quelques questions étudiées dans le cadre du projet Steve : Les annotations des utilisateurs diffèrentelles des termes employés dans la documentation par les professionnels des musées? Les membres du personnel des musées trouvent-ils utiles les annotations des utilisateurs? Les annotations des utilisateurs permettront-elles d'améliorer la recherche (une raison souvent citée pour lappui aux annotations produites par les utilisateurs pour les collections muséales en ligne) ? On a découvert, entre autres, dans le cadre du projet Steve que le vocabulaire employé par le public était différent de celui employé dans les musées (Trant, 2009).

Les chercheurs de la communauté Interaction personne-machine ont fait état de la similitude des 
Les annotations par le public et les folksonomies pouvaient contribuer de façon positive à l'accessibilité des collections en ligne des musées des beauxarts en plus d'être une activité engageante pour certains visiteurs en ligne.

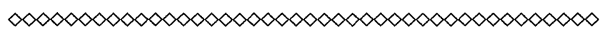

processus cognitifs entre la production de mots clés pendant l'annotation par les utilisateurs individuels et la production de mots clés lors de la recherche, ce qui suggère le potentiel positif des projets liés à l'annotation (Furnas et coll., 2006). Le projet Steve n'a pu déterminer à partir des journaux de recherche pour quelles œuvres les visiteurs en ligne faisaient de la recherche dans les sites Web des musées; par conséquent, il n'est pas possible de répondre directement à la question visant à savoir si les annotations produites par les utilisateurs obtiennent de meilleurs résultats de recherche. On a pu établir que les termes attribués correspondaient aux termes des journaux de recherche dans 22,6 à 38,5\% des cas pour les deux établissements étudiés. On a conclu que les annotations par le public et les folksonomies pouvaient contribuer de façon positive à l'accessibilité des collections en ligne des musées des beaux-arts en plus dêtre une activité engageante pour certains visiteurs en ligne (Trant, 2009).

Découverte intéressante du projet Steve, 11 des 1691 utilisateurs ont contribué à plus de $1 \%$ du nombre total d'annotations pour un total de $20,4 \%$ de l'ensemble des annotations (Trant, 2009). De tels annotateurs très actifs sont présents également dans d'autres projets, notamment The Commons de Flickr. Digg, un site populaire de recommandations d'actualités, a découvert que les dix premiers utilisateurs de Digg dominaient la page d'accueil en présentant des milliers de liens quotidiennement; ils ont été bannis par la suite, mais cet exemple montre le potentiel de compétition lorsque des expéditeurs actifs souhaitent figurer en haut d'une liste (Ruberg, 2008). Ce que la présence des ces utilisateurs hyperactifs suggère relativement au contenu muséal, c'est que bien que louverture du contenu au grand public élargisse la possibilité de "parole» offerte, seule une petite population pourrait malgré tout être entendue.

Au cours de l'année 2007, le Musée McCord a ajouté à son site Web une section officielle intitulée «Mon McCord » ainsi que des nouvelles fonctions d'utilisation des ordinateurs à des fins sociales, notamment un espace pour les annotations, un jeu d'annotations, une fonction permettant aux utilisateurs inscrits d'ajouter aux images proposées des commentaires, une fonction permettant aux utilisateurs inscrits de partager immédiatement leurs circuits Web et une fonction qui permet aux utilisateurs

\section{Figure 2}

Espace personnalisé, Musée McCord (Montréal, QC).

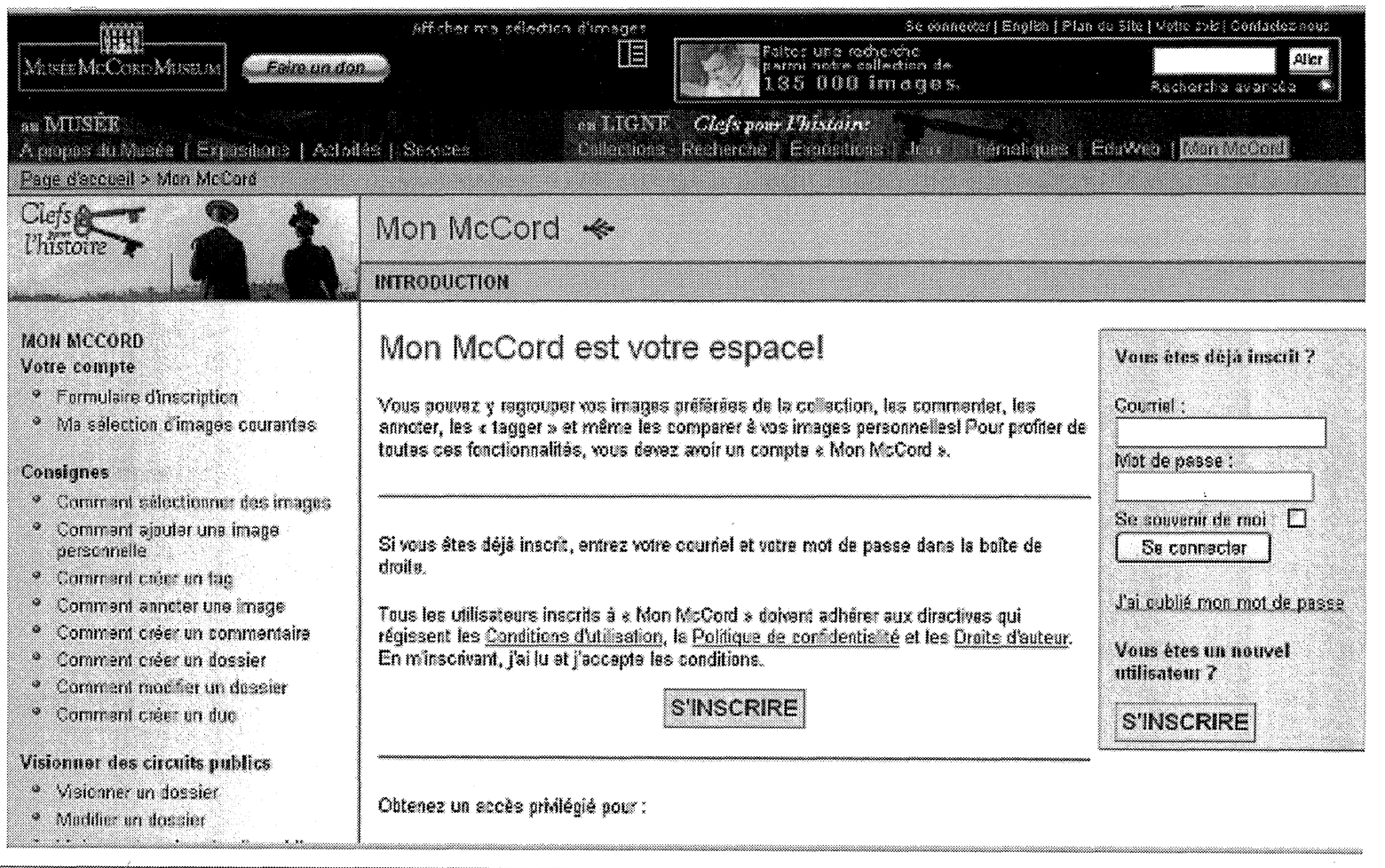


inscrits d'annoter les images (RCIP, 2008). Le Musée McCord a évalué les risques suivants liés au projet :

1. risque technique (capacité de mettre en place et de gérer les fonctions) : minime. - Le Musée McCord possède l'expertise technique nécessaire à l'interne ainsi qu'une connaissance approfondie de son propre site Web ;

2. risque financier (frais de mise en place et d'entretien des fonctions) : minime. Les fonds externes ont servi à la mise en place de ces fonctions ;

3. risque sur le plan des ressources humaines (exigence de compétences de gestion et techniques) : minime. - Les compétences à l'interne sont suffisantes ;

4. risque concernant la publication des résultats (adoption des fonctions) : une période pouvant aller jusqu'à trois ans pourrait s'écouler avant d'obtenir suffisamment de données pour déterminer l'incidence d'une fonction donnée ;

5. risque sur le plan légal ou politique (responsabilité, droits d'auteur, confidentialité des données, protection de la vie privée des mineurs, etc.) : minime. - Le Musée McCord maîtrise tous les aspects des droits d'auteur portant sur les images (RCIP, 2008). Aucune permission supplémentaire n'est nécessaire pour diffuser du contenu dans son site Web. Par ailleurs, les institutions et les personnes à qui une licence Creative Commons a été octroyée ont le droit d'utiliser ce matériel à des fins non commerciales.

Tous les autres risques, notamment les risques sur les plans légal et politique qui encadrent les fonctions Web 2.0, sont les mêmes que ceux envisagés par d’autres institutions aux prises avec des décisions semblables. On traite de ces préoccupations en essayant dêtre aussi au courant et responsable que possible, sans être trop restrictif. Le Musée McCord fait son possible pour se tenir au courant de la législation en vigueur de temps à autre en la matière.

Depuis l'introduction le 9 août 2007 du jeu des annotations dans le site du Musée McCord, plus de 215000 annotations ont été recueillies. Les données ont été peu concluantes étant donné que le Musée McCord ne fait pas la promotion de ses lancements; une période allant jusquà trois ans pourrait sécouler avant que l'incidence réelle d'une fonction soit connue.

Parmi les autres risques cernés, mentionnons celui de faire croire à tort que le contenu social constitue le contenu officiel. Ce risque est important pour les musées et il est essentiel que les fonctions qui permettent au public de formuler des commentaires fassent la différence entre le contenu officiel du musée et les annotations ajoutées par les utilisateurs. Les musées sont considérés comme des entités fiables et les visiteurs les apprécient pour cette raison; donc, le contenu ajouté par le public
Parmi les autres risques cernés, mentionnons celui de faire croire à tort que le contenu social constitue le contenu officiel.

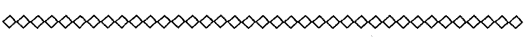

doit être distingué du contenu officiel du musée. En fait, la question a été soulevée récemment avec un groupe d'étudiants de troisième année en Histoire de l'Université Carleton, à Ottawa. Les étudiants ont déclaré haut et fort qu'ils devaient être capables d'identifier le contenu du musée et que, dans certains cas, ils ne voulaient même pas consulter le contenu ajouté par "n'importe qui». L’enquête "Les Canadiens et leurs passés " $(<\mathrm{http}$ ://www. cshc.ubc.ca/Canadians_and_Their_Pasts.pdf $>$ ) a révélé que sur les 3419 réponses à la question "Les musées sont-ils fiables?» (How trustworthy are museums?), $63,35 \%$ des personnes interrogées ont répondu «Très fiables », et $29,8 \%$ ont répondu «Assez fiables », tandis que seulement $2,1 \%$ ont répondu soit « Pas tellement » ou «Pas du tout fiables", ce qui indique que les musées sont considérés comme des sources dignes de foi, et il est important que cette réputation soit maintenue (Northrup et Létourneau, 2009).

\section{Participation de la communauté dans l'élaboration des histoires}

Finalement, les musées peuvent permettre aux visiteurs de contribuer au contenu de leurs expositions en ligne. Une analyse des 1 ooo messages de commentaires reçus dans le cadre des expositions Histoires de chez nous du Musée virtuel du Canada (MVC) (<http:// www.museevirtuel-virtualmuseum.ca/ $>$ ) a permis de les classer en fonction des types suivants :

- partage d'information ou commentaires à partir du site;

- question, demande d'information supplémentaire ou communication d'une erreur ;

- bon nombre de visiteurs connaissent quelqu'un présenté dans le site ;

- les visiteurs souhaitent contribuer à l'information, aux ressources ;

- questions liées à la recherche généalogique.

Bien que plusieurs des messages constituaient des demandes de renseignements supplémentaires de la part d'un visiteur ayant entrepris une recherche généalogique, plusieurs messages provenaient de visiteurs offrant une photo ou un document pour contribuer à l'exposition, partageant de l'information supplémentaire qu'ils souhaitaient voir ajoutée dans le site, ou communiquant au personnel du musée des renseignements qu'il devait connaître à leur avis. Ces messages ont circulé 


\section{En outre, l'établissement doit être à l'aise avec les politiques des sites externes puisque les utilisateurs sont tenus de se conformer aux politiques de ces sites en matière de contenu.}

en coulisse, mais ils ajoutent un élément social au site et permettre aux visiteurs de contribuer de cette façon, en communiquant de l'information parfois inestimable, devrait faire partie intégrante de la prochaine version des Histoires de chez nous. En fait, c'est un bon exemple d'utilisation par les musées des fonctions liées aux médias sociaux dans leurs propres sites, particulièrement lorsqu'ils sollicitent la participation d'une communauté en particulier.

Sur le site Moving Here (<http://movinghere.org. $\mathrm{uk}>$ ), on recueille les histoires des immigrants installés au Royaume-Uni. Le partenariat Moving Here est dirigé par les Archives nationales, et il comprend un consortium de 30 organismes d'archivage, bibliothèques et musées qui ont contribué à la collecte du matériel d'archives du site. Le site présente une galerie, des histoires de migration organisée, certains documents d'archives visant à aider les gens à retracer les itinéraires et, finalement, la section comportant les histoires. De tels projets communautaires, où l'apport de la communauté est activement sollicité et contribue fortement à alimenter le thème, sont des exemples impressionnants de ce que l'on peut réaliser en mettant en œuvre des applications de médias sociaux.

Le Reciprocal Research Network (RRN) est un réseau de recherche axé sur la technologie réunissant des communautés, des chercheurs et des établissements culturels. Il permet à des utilisateurs et à des établissements éloignés sur le plan géographique de poursuivre des projets de recherche individuels ou collectifs en matière de patrimoine culturel ( $<$ http://www.moa.ubc. ca/RRN/about_overview.html $>$ et $<$ http://www.rrnpilot. org/login $>$ ). Les utilisateurs ouvrent une session et explorent la base de données. Ils ont la possibilité de discuter entre eux, de suggérer des changements concernant un enregistrement muséal et d'utiliser un "bloc adhésif » pour consigner des notes et des observations personnelles. Susan Rowley, conservatrice du musée et professeure d'anthropologie à l'Université de la ColombieBritannique (UBC), affirme que les collections de musées ont toujours été rassemblées par des étrangers qui se rendaient dans les collectivités et recueillaient des objets, en particulier certains des premiers explorateurs qui visitaient un lieu une journée, recueillaient des objets et les rapportaient avec eux. "Ainsi, ce qui intéresse les communautés, c'est de relier les objets à la langue, à la terre, aux histoires ", confie-t-elle (Webb, 2008).
Un autre projet, encore plus axé sur la participation du public, est le site introduit par le Tate Museum de Liverpool intitulé The One that Spoke to $\mathrm{Me}(<\mathrm{http}: / /$ totstm.tate.org.uk $>$ ). Ce site affiche une sélection des œuvres d'art du XXe siècle pour lesquelles il est possible de lire les commentaires formulés par les autres visiteurs, de visionner une vidéo de leurs réactions aux œuvres, d'explorer les objets ou de communiquer son opinion sur les œuvres. Ce n'est qu'après avoir pris connaissance des renseignements fournis par le public que l'on accède à l'enregistrement de conservation des œuvres d'art. Cette façon de faire va à lencontre du mode de diffusion d'information de la plupart des sites muséaux, mais cétait précisément le but de ce projet. Autre initiative dans le cadre du projet, quatre œuvres d'art ont été disposées à divers endroits de Liverpool, où les gens sont invités à livrer leurs commentaires à leur sujet.

\section{Conclusion}

Différents musées expérimenteront divers degrés de confort avec les applications de médias sociaux et ils devront déterminer celles qui s'harmonisent le mieux avec la mission de leur établissement, avec leur « personnalité » et la situation de leur personnel. Des organismes aussi différents que le Brooklyn Museum et le Powerhouse Museum ont mis en application des initiatives prometteuses, souvent dirigées par des individus énergiques. Certains organismes qui s'adressent à une clientèle particulière ou qui projettent une image organisationnelle plus «expérimentale» ou "de plaisir» (par exemple, les centres des sciences) pourraient jouir d'une plus grande liberté pour expérimenter les applications des médias sociaux par rapport à d'autres établissements qui cultivent une image plus classique et s'adressent à un public plus conservateur. En outre, létablissement doit être à l'aise avec les politiques des sites externes puisque les utilisateurs sont tenus de se conformer aux politiques de ces sites en matière de contenu. Ils doivent être prêts à assumer le risque associé au fait de donner aux utilisateurs la possibilité d'utiliser le contenu muséal sans autorisation, parfois d'une façon que le musée n'approuverait pas et hors de son contexte. Par ailleurs, la mesure selon laquelle un établissement prévoit assurer une surveillance aura également une incidence sur les projets qu'un musée sera prêt à entreprendre.

Comme lors des débuts d'Internet, à lépoque où les musées se questionnaient à savoir si la publication de leurs collections en ligne aurait un effet négatif sur lachalandage des visiteurs dans leur établissement, les musées doivent analyser la part de l'effort qui doit être consacrée à la création d’applications qui profiteront en bout de ligne uniquement aux visiteurs en ligne et aux visiteurs imprévus qui tombent par hasard sur le contenu en consultant un autre site. C'est une question d'équilibre entre les risques et les avantages, les attentes des utilisateurs et la capacité du musée. Les musées doivent 
prendre soin d'abord et avant tout des collections dont ils ont la garde, tout en demeurant pertinents à une époque où les visiteurs s'attendent à pouvoir interagir avec le contenu, et ils doivent également savoir reconnaitre quelles sont les activités qui sont avantageuses en élargissant la portée du musée. $\odot$

\section{Sources consultées}

Sauf exception, les pages Web ont été consultées sur Internet le 30 avril 2009.

Alexander, C., et al. 2008. Beyond Launch : Museum Videos on YouTube. J. Trant and D. Bearman (eds.). Museums and the Web 2008 : Proceedings, Toronto : Archives \& Museum Informatics. Published March 31, 2008. <http://www.archimuse. $\mathrm{com} / \mathrm{mw} 2008 /$ papers/hart/hart.html $>$.

Bernstein, S. 2008. Where Do We Go From Here : Continuing with Web 2.0 at the Brooklyn Museum. J. Trant and D. Bearman (eds.). Museums and the Web 2008 : Proceedings. Toronto : Archives \& Museum Informatics. Published March 31, 2008. $<$ http://www.archimuse.com/mw20o8/papers/bernstein/ bernstein.html>.

Bray, P. 2009. Open Licensing and the Future for Collections. J. Trant and D. Bearman (eds). Museums and the Web 2009: Proceedings. Toronto : Archives \& Museum Informatics. Published March 31, 2009. <http://www.archimuse.com/ mw20og/papers/bray/bray.html $>$.

Budiu, R., P. Pirolli, L. Hont. 2009. Remembrance of Things Tagged; How Tagging Effort Affects Tag Production and Human Memory. CHI 2009, Boston.

Cary, W. 2008. Introducing 1stfans : A Socially Networked Museum Membership. <http://www.brooklynmuseum.org/community/blogosphere/bloggers/2008/12/05/introducing-1stfansa-socially-networked-museum-membership/> (consulté sur Internet le 22 avril 2009).

Chan, S. 2006. Powerhouse Museum Launches Web 2.0-Styled Collection Search. <http://www.powerhousemuseum.com/dmsblog/ index.php/2006/06/o8/powerhouse-museum-launches-web20-styled-collection-search/>.

Chan, S. 2007. A Reminder about User Incentives. <http:// www.powerhousemuseum.com/dmsblog/index. php/2007/06/27/a-reminder-about-user-incentives/>.

Chan, S. 2008a. Commons on Flickr: A Report, Some Concepts and a FAQ : the first 3 Months from the Powerhouse Museum. <http:// www.powerhousemuseum.com/dmsblog/index.php/2008/07/21/ commons-on-flickr-a-report-some-concepts-and-an-faq-thefirst-3-months-from-the-powerhouse-museum/>.

Chan, S. 2008b. Re-ingesting Flickr tags from the Commons back into our collection OPAC. <http://www.powerhousemuseum.com/ dmsblog/index.php/2008/07/25/re-ingesting-flickr-tags-fromthe-commons-back-into-our-collection-opac/>.

Chan, S. 2009. One Year in the Commons on Flickr statistics and a book. http://www.powerhousemuseum.com/dmsblog/index.php/2009/04/o8/ one-year-in-the-commons-on-flickr-statistics-and-a-book/>.

Ferrante, R. 2009. Moving beyond us vs, them. <http://smithsonian20.typepad.com/blog/2009/o3/moving-beyond-us-vsthem.html> (consulté sur Internet le 22 avril 2009).

Furnas, G.W., et al. 2006. Why do tagging systems work ? Extended Abstracts CHI 2006, ACM Press. 36-39.

Griffiths, J. W., and D. W. King. 2008. Interconnections : The IMLS Study on the Use of Libraries, Museums, and the Internet, February 2008. <http://interconnectionsreport.org/reports/ ConclusionsSummaryFinalB.pdf $>$.

Griffith, M. 2007. Pew Internet and American Life Project Hobbyists Online, September 19, 2007. <http://pewresearch.org/pubs/597/ hobbyists-online $>$.
Henry, D. 2007. The few, the proud : the Wikipedia authors. <http:// www.mndaily.com/2007/11/13/few-proud-wikipedia-authors $>$ (consulté sur Internet le 22 avril 2009).

ICOM Statutes art.3.para.1. ICOM Definition of a Museum. <http:// icom.museum/definition.html> (consulté sur Internet le 22 avril 2009).

Jones, S., and S. Fox. 2009. Generations Online in 2009. Pew Internet and American Life Project. <http://www.pewinternet.org/ Reports/2009/Generations-Online-in-2009.aspx >.

Lenhart, A. 2009. Adults and Social Network Websites. <http://www. pewinternet.org/Reports/2009/Adults-and-Social-NetworkWebsites.aspx>.

Marsan, C. 2009. Ten Twitter Tips from Early Federal Adopters. NetworkWorld. <http://www.networkworld.com/ news/2009/040909-feds-twitter-tips.html ?page $=1>$.

McKenzie, B. 2008. Flickr and Museums. <http://bridgetmckenzie. blogspot.com/2008/o7/flickr-and-museums.html> (consulté sur Internet le 22 avril 2009).

Global Faces and Networked Places: A Nielsen report on Social Networking's New Global Footprint. 2009. <http://blog.nielsen. com/nielsenwire/wpcontent/uploads/2009/03/nielsen_globalfaces_maro9.pdf $>$.

Northrup, D., and J. Létourneau. 2009. Canadians and Their Pasts. Institute for Social Research, York University and le Centre interuniversitaire d'études sur les letters, les arts et les traditions (CELAT), Université Laval. Presentation to the Canadian Museums Association. Toronto, Ontario, March 27, 2009.

Pew Internet and American Life Project. 2009. Pew Internet Trend data. <http://www.pewinternet.org/Data-Tools/DownloadData/Trend-Data.aspx> (consulté sur Internet le 22 avril 2009).

Réseau canadien d'information sur le patrimoine. 2004. Enquête 2004 auprès des visiteurs des musées et de l'espace Internet des musées : Documentation et résultats. <http://www.pro.rcip. $\mathrm{gc.ca} />$ (consulté sur Internet le 3 septembre 2009).

Réseau canadien d'information sur le patrimoine. 2008. L'utilisation des ordinateurs à des fins sociales à partir du site Web du Musée McCord. <http://www.pro.rcip.gc.ca> (consulté sur Internet le 3 septembre 2009).

Rubert, B. 2008. Digg's top users banned for ever and ever. <http://www.sfweekly.com/2008-10-01/news/ digg-s-top-users-banned-forever-and-ever/>.

Simon, N. 2008a. Museum 2.0: The future of Authority: Platform Power. <http://museumtwo.blogspot.com/2008/10/futureof-authority-platform-power.htm $>$ (consulté sur Internet le 22 avril 2009).

Simon, N. 2008b. Museum 2.0 : What's a Virtual Visitor Worth? $<$ http://museumtwo.blogspot.com/2008/12/whats-virtualvisitor-worth.html> (consulté sur Internet le 22 avril 2009).

Springer, M., et al. 2008. For the common good: The Library of Congress Flickr pilot project. <http://www.loc.gov/rr/print/ flickr_report_final.pdf > (consulté sur Internet le 7 septembre 2009).

Trant, J. 2009. Tagging, Folksonomy and Art Museums: Results of Steve.Museum's Research. <http://conference.archimuse. com/files/trantSteveResearchReport2008.pdf> (consulté sur Internet le 22 avril 2009).

Von Appen, K., et al. 2009. WeTube : Getting Physical with a Virtual Community at the Ontario Science Centre. J. Trant and D Bearman, (eds) Museums and the Web Proceedings. Toronto : Archives \& Museum Informatics. $<$ http://www.archimuse. com./mw2009/papers/vonappen/vonappen.html>.

Von Ahn, L., and L Dabbish. 2004. Labeling Images with a Computer Game. CHI, Vienna, 2004.

Webb, J. 2008. Native artifacts go digital at UBC. $<$ http://www. straight.com/article-158380/native-artifacts-go-digital $>$.

Yasko, J. 2007. Museums and Web 2.0, 2007. <http://ww.aam-us.org/ pubs/mn/museumsweb.cfm>. 